\title{
Automated Brain Tumor Detection Based on Feature Extraction from The MRI Brain Image Analysis
}

\author{
Ban Mohammed Abd Alreda*, Hussain Kareem Khalif, Thamir Rashed Saeid \\ Electrical Engineering Department, University of Technology, Iraq
}

*Ban Mohammed Abd Alreda

Electrical Engineering Department,

University of Technology, Iraq

Email: 31630@student.uotechnology.edu.iq

\begin{abstract}
The brain tumors are among the common deadly illness that requires early, reliable detection techniques, current identification, and imaging methods that depend on the decisions of neuro-specialists and radiologists who can make possible human error. This takes time to manually identify a brain tumor. This work aims to design an intelligent model capable of diagnosing and predicting the severity of magnetic resonance imaging (MRI) brain tumors to make an accurate decision. The main contribution is achieved by adopting a new multiclass classifier approach based on a collected real database with new proposed features that reflect the precise situation of the disease. In this work, two artificial neural networks (ANNs) methods namely, Feed Forward Back Propagation neural network (FFBPNN) and support vector machine (SVM), used to expectations the level of brain tumors. The results show that the prediction result by the (FFBPN) network will be better than the other method in time record to reach an automatic classification with classification accuracy was $\mathbf{9 7 \%}$ for 3-class which is considered excellent accuracy. The software simulation and results of this work have been implemented via MATLAB (R2012b).
\end{abstract}

KEYWORDS: Feed forward back propagation neural network, Support vector machine, Discrete wavelet transform, Texture features by gray level co-occurrence matrix (GLCM).

\section{INTRODUCTION}

Research in the domain of biomedical image analysis has been one of the most challenging and promising areas in recent years due to an increase in brain tumors. Sadly, there are many of these tumors are discovered late time when symptoms of the disease are present and when the tumor has become large, making it very difficult to treat or remove the tumor and dangerous. While it is much safer and easier for the removal of a limited tumor at an early stage. Approximately 60 percent of glioblastomas (known as glioblastoma multiform GBM) begin as lower-grade tumors but become large tumors over time [1]. Computer-assisted surgical preparation and modern image-guided systems have been widely used in neuron surgery [1]. Therefore, when the development of medical devices in general and medical imaging devices in particular, many types and different methods of medical imaging have appeared recently. So there are many types of imaging are used in brain tumors diagnoses such as magnetic resonance imaging (MRI), radiography (like CT and $\mathrm{x}$-ray), and ultrasound imaging [2] . Therefore, the diagnosis of the tumors in the brain are based on image analysis of the MRI, and it is a saver method and doesn't affect the human body because it doesn't use any radiation anymore. It's based on the magnetic area and radio waves [2]. The primary reason for death in kids and adults is the brain tumor [3,4]. The survival chances may be higher if the tumors are correctly detected at an early stage $[3,4]$.

\section{LITERATURE SURVEY}

The need for an automated and well-organized brain tumor MR Image Classification and Diagnosis system has increased with accurate results for proper treatment directions (therapy and surgery planning). To this purpose, a great several research has been submitted by different researchers with good results but some researchers did not use real data with data taken from the Internet, and no more features of the brain images were extracted that would therefore be used as inputs for the classifiers used. This section will conclude with a summary discussion of previous.

Naveena H. S. et al. [5] Utilize ANN ability to classify MRI images as either cancerous or non-cancer tumors. Segmentation is achieved by the K-means clustering algorithm. The Gray Level Co-Occurrence Matrix (GLCM) was then used to extracted features from the segmented image. Finally, the classification of brain tumors was performed by the Back Propagation Neural Network (BPNN) and the Probabilistic Neural Network (PNN) with a system accuracy of $79.02 \%$ and $97.25 \%$ respectively.

This is an open access article under the terms of the Creative Commons Attribution License, which permits use, distribution and reproduction in any medium, provided the original work is properly cited.

(C) 2020 The Authors. Published by Iraqi Journal for Electrical and Electronic Engineering by College of Engineering, University of Basrah. 
Ahmad Chaddad and Camel Tanougast [6] The proposed algorithm uses a contrast enhancement image in preprocessing and segmentation using the proposed adaptive skull stripping algorithm and detection tumor based entirely on multi-threshold segmentation (MTS) to identify characteristic pixel proximity by MTS algorithm threshold detects.

Bahadure et. al. [7] Proposed BWT and SVM imaging techniques for MRI-based brain tumor detection and classification. In this method, $95 \%$ accuracy was achieved by SVM and segmentation by skull stripping, which eliminated all non-brain tissues for detection purposes.

N. Varuna Shree and T. N. R. Kumar [8] region-based segmentation images feature extraction 7 features from GLCM. MRI images of $256 \times 256,512 \times 512$-pixel size on dataset. dataset collected from Web sites www.diacom.com. The accuracy of training $100 \%$ dataset because the statistical textural features were extracted from LL and HL sub bands wavelet decomposition and 95\% of testing dataset.

Parasuraman Kumar and B. Vijay Kumar [9] FCM Clustering Algorithm based segmentation and feature extraction by using GLCM and Gabor are 9 features. Preprocessing achieved by median filter. Ensemble methods combine the procedure of feed forward neural network, extreme learning machine (ELM) and support vector machine classifiers to detected tumor. The accuracy for ensemble classifier is $91.17 \%$ whereas FFANN, ELM and SVM have an accuracy of $84.33 \%, 86 \%$ and $89.67 \%$ respectively.

Ahsanullah Umary and Harpreet Kaur [10] The suggested approach includes eliminating unnecessary noise using filtering techniques, segmenting by using the threshold to remove the skull from the image, utilizing GLCM to extraction characteristics and classification by applying SVM to tumor and non-tumor image. This proposed approach was approximately $90 \%$ accurate.

\section{Proposed Methodology}

The work proposed, contribute with the help of Nonspecialists to the understanding of the diagnostic mechanism and will be contribute diagnosis decision whether the tumor is present or absent along with the exact area of the tumor. According to the proposed work stages as shown in Fig. 1.

\subsection{Data acquisition stage}

Harvard medical brain database most widely available on the internet used by many researchers, will be used in this work to test proposed algorithm in the classify the tumor of the brain using actual data which are 83 cases for training and 37 cases for testing. Also, data from the cancer imaging archive (TCIA) 59 casesfor training and 29 cases for testing, as well through fieldwork to collecte 66 cases for training and 34 cases for testing from General Hospital of Baquba-Iraq of MRI brain images. So, it becomes some of the data based in this work 308 images using T1 weighted and T2-weighted images. The MRI brain images data that taken from available Harvard medical brain databases were available in the form of images and were directly used for the following preprocessing stage. While, the MRI brain images data taken from hospitals was in the form of CD where was MRI brain images in DCM format (real MRI brain images data) [11,12], and by using an online website for image format conversion and saved in the form of digital color images at format types JPEG in the computer [13].

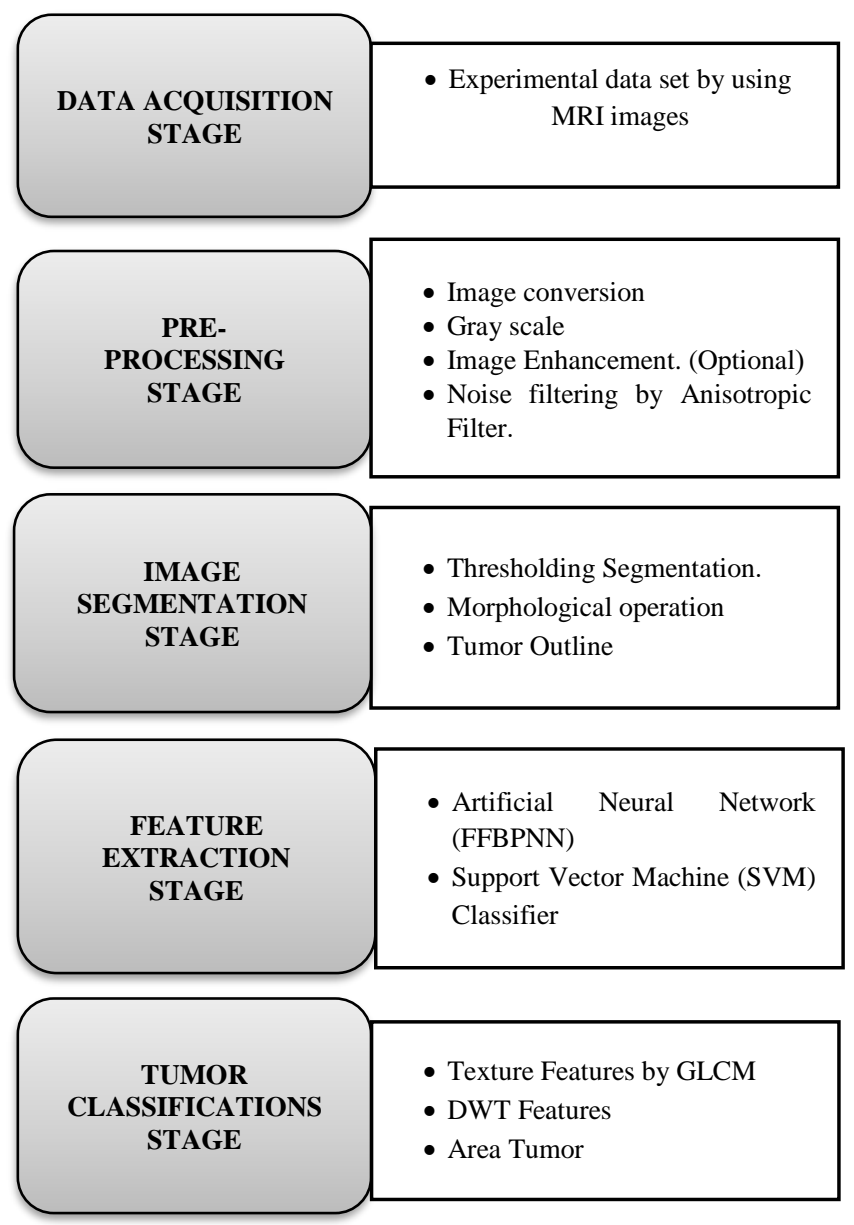

Fig. 1: Flowchart of the proposed methodology.

\subsection{Pre-processing stage}

The main task of pre-processing is to enhance the precision of the MR images and also to get it suitable for further processing by a human or machine vision system. The grayscale image is quite important for many purposes, such as segmentation of images, extraction of features and classification of images using the (rgb2gray) function of the MATLAB software used to convert the RGB image to the Grayscale images [14]. The MATLAB (adjust) function has been used to increase the Contrast some image by assigning the values of the input intensity of the image to new values such that the information is saturated at low and high input data level [15].

Sometimes MRI images contain noise that must be discarded and removed. The causes of this noise are due to the high frequency of radio waves and the patient's movement during the MRI [15]. But the noise removal should not destroy the edges of the image and reduce the clarity and quality of the image. Anisotropic filtering used anisotropic diffusion filter is an image filtering method proposed by Persona and Malik $[16,17]$. The anisotropic diffusion filter is the spearheading work in partial derivatives equations (PDE) based de-noising 
$[17,18]$. The algorithm is implemented on the MATLAB software used function (anisodiff.m) [19]. The remaining steps of the methods are explained below.

The Algorithm

1) Load gray MRI images into the MATLAB environment.

2) Convert into Double precision.

3) Add the random noise to the image.

4) Apply to open by reconstruction

5) Give the number of iterations $(\mathrm{i}=\mathrm{N})$

6) $i=1$

7) Enter the following value - Constant integration. (Usually, this parameter is set to its maximum value due to numerical stability).

- The gradient modulus threshold that controls the conductivity.

- Functions of the conduction coefficient (proposed by Perona \& Malik) Apply anisotropic diffusion filtering process.

8) if $(\mathrm{i}<\mathrm{N}) \mathrm{i}=\mathrm{i}+1$ go to step no:7.

9) Display gray input image and filtered output.

\subsection{Image segmentation stage}

The segmentation technique plays a significant role in the processing of images. Segmentation results will be used to obtain quantitative information from images, including clustering, thresholding, etc.

\subsubsection{Threshold segmentation}

Threshold segmentation technology Plays an essential part in the processing of images. The threshold segmentation is used to extract the various regions from the whole image according to the difference in intensity. There are many different methods to achieve thresholding methods, such as the Otsu method [20]. Otsu's method is one of the most effective methods used for image thresholding. It is based on the criterion of minimizing class variance; however, the search for the global optimal threshold is a fully comprehensive algorithm. At first, the Otsu method needs to calculate a gray-level histogram [21].

$\sigma_{\omega}^{2}(t)=\omega_{1}(t) * \sigma_{1}^{2}(t)+\omega_{2}(t) * \sigma_{2}^{2}(t)$

$\omega i$ Weights are The probability of the two classes divided by the thresholds $t$ and $\sigma i 2$ variances of these classes.

$$
\begin{aligned}
\sigma_{b}^{2}(t) & =\sigma^{2}-\sigma_{2}^{2}(t) \\
& =\omega_{1}(t) * \omega_{2}(t) *\left[\mu_{1}(t)-\mu_{2}(t)\right]^{2}
\end{aligned}
$$

Which is described in terms of class probabilities $\omega i$ and class means $\mu_{1}$. The class probability $\omega_{1}(t)$ is calculated from the histogram as $\mathrm{t}$ :

$\omega_{1}(t)=\sum_{0}^{t} p(i)$

Whereas the class mean $\mu_{1}(t)$ is:

$$
\mu_{1}(t)=\left[\sum_{0}^{t} p(i) * x(i)\right] / w_{1}
$$

In which $(i)$ is the value at the center of the bin $i_{t h}$ histogram. Fig. 2 show steps of proposed algorithm in the work.

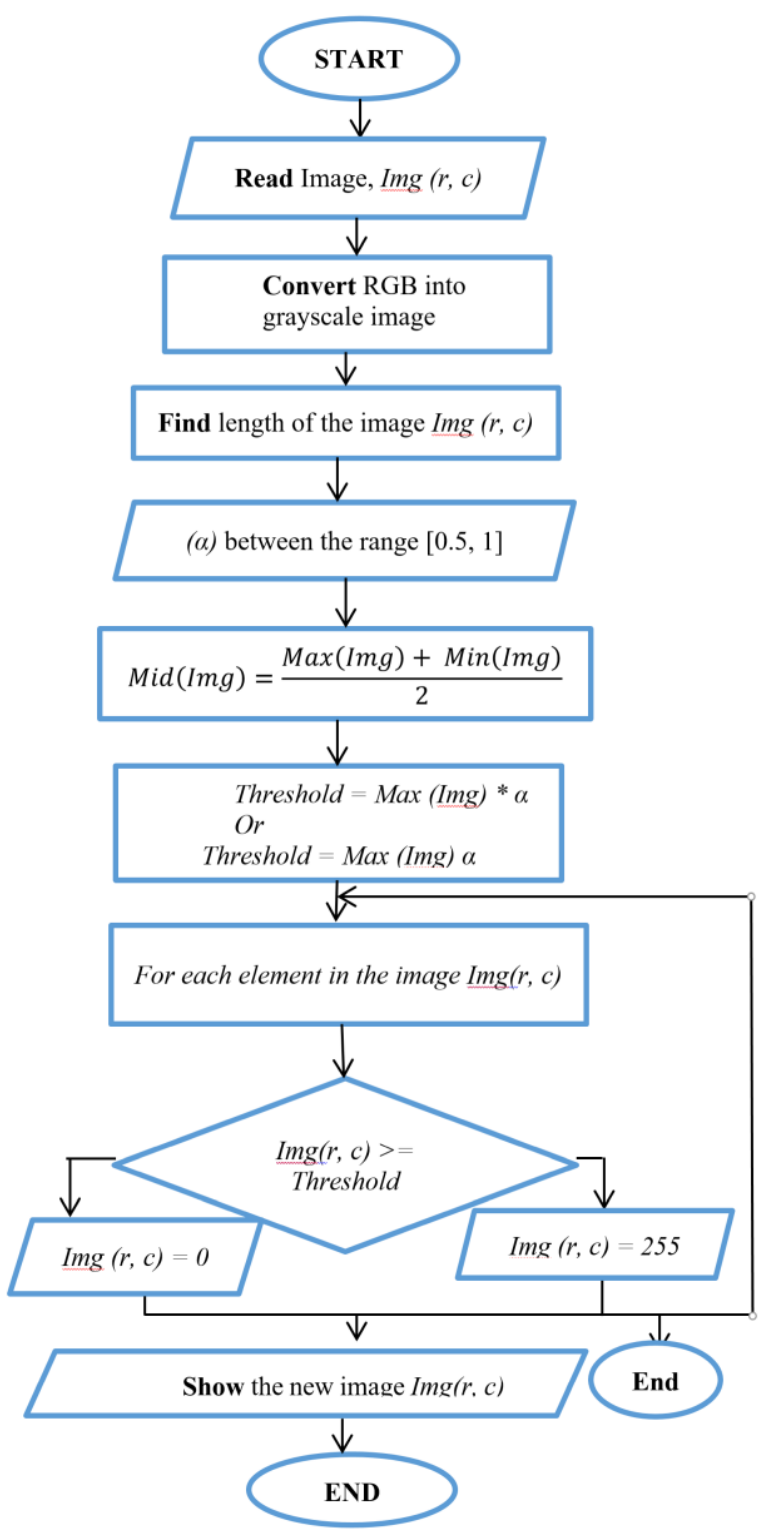

Fig. 2: Flowchart of proposed algorithm

\subsubsection{Morphological operation}

Typically, applied on the binary images (black \& white images) where the pixels value is between 0 and 1 . This work is used in post-processing to enhance the threshold segmentation by removing noise and to filter out smaller areas. The first step is very important to remove undesired pixels as noise by filling the holes that can be described as a distortion in the image. Where the small holes fill the white pixel in the dark background by dark pixel and the holes dark in the white region will convert to a white pixel. Filling region algorithm based on set dilations, complements, and intersections. In the second step, erosion operations are intended to eliminate pixels from the boundary area of the objects. The operation of adding or deleting pixels to or from the boundary area of the object is focused on the structuring elements of the selected image. 


\subsubsection{Tumor Outline}

Tumor outline is an additional step used to determine the shape of the tumor and external limits. Image pixel subtraction operators take two images as an input of the same size and produce as output a third image; whose pixels values are the values obtained by subtraction between the two images. The pixel subtraction operation can be defined as [22,23]:

$\operatorname{Img}_{3}(r, c)=\operatorname{Img}_{1}(r, c)-\operatorname{Img}_{2}(r, c)$

Where $\operatorname{Img}_{3}(r, c)$ represented the result of subtracting the two images, $\operatorname{Img}_{1}(r, c)$ represented the result of the process of segmentation of the image to $\mathrm{BW}$ by the threshold segmentation algorith, $\operatorname{Img}_{2}(r, c)$ represented the resulting image of the erosion process as show in Fig. 3.

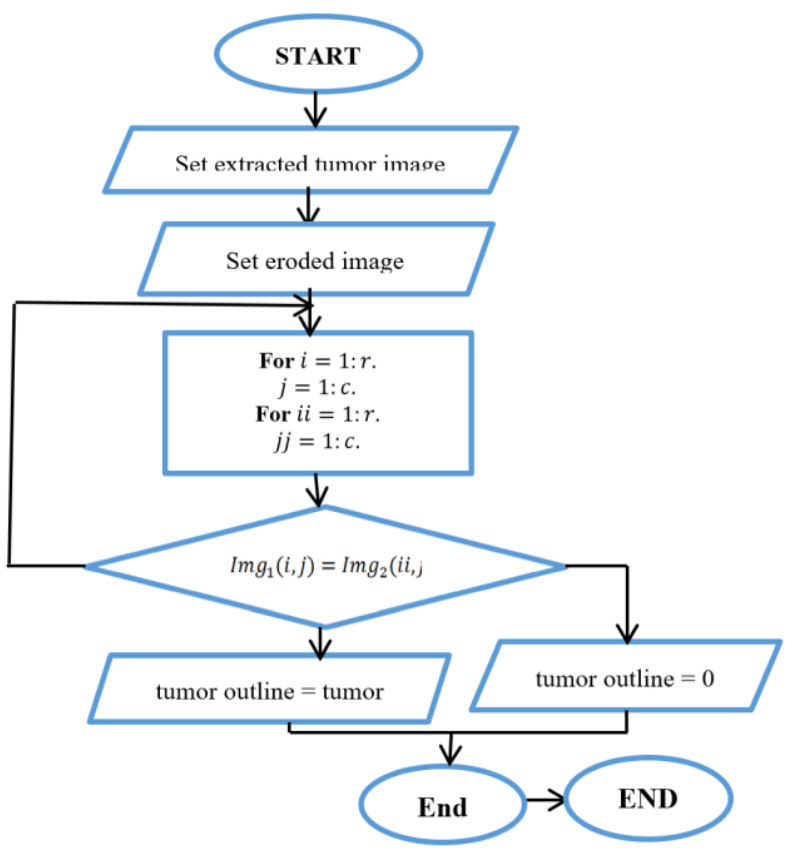

Fig. 3: flowchart of tumor outline algorithm

\subsection{Feature extraction stage}

Extracting features is to extract and transform details for input information into several features, called a feature vector, by decreasing the pattern of data representation. The components set will obtain the extracted from the input data (image) to execute the classification task.

\subsubsection{Discrete wavelet transform (DWT)}

DWT has the benefit of extracting the most appropriate features of various paths and dimensions as it provides local signal time-frequency details utilizing cascaded high-pass and low-pass filter banks to features extracted in a hierarchical structure [24]. As a consequence, there are four sub-band images at each point (LL, LH, HH, HL). Approximate components of the image may represent the LL sub-band, while the LH, HL, HH sub-bands may be considered as the accurate components of the image [24,25]. Fig. 4 shown DWT 2-level decomposition of the image. The different frequency components and each component were studied with a resolution matched to its scale and expressed as:

$D W T P(u)\left\{\begin{array}{l}d_{i, j}=\sum p(u) H * i(u-2 i j) \\ d_{i, j}=\sum p(u) G * i(u-2 i j)\end{array}\right.$
The coefficients di,j refers to the component attribute in signal $\mathrm{p}(\mathrm{u})$ corresponding to the wavelet function, whereas bi,j refers to the approximated components in the signal. The functions $\mathrm{h}(\mathrm{u})$ and $\mathrm{g}(\mathrm{u})$ in the equation represent high-pass and low-pass filter coefficients, respectively, while parameters $\mathrm{i}$ and $\mathrm{j}$ refer to wavelet scale and translation factors. In simulated images, the following wavelets were used: Symlet 7 (sym7), Daubechies 4 (db4), Hear (haar), Biorthogonal 3.5, and 3.7 (bior 3.5 and bior 3.7) respectively. These wavelets were used to compare and find which level of decomposition and which wavelet produces better results. In this work, a 2-level decomposition of (sym) wavelet is used to derive 12 features for each brain MRI. In this work, various statistical features are calculated such as mean, standard deviation, median, range, mode, max, min, etc. In this step, the features extracted from the gray image are based on a histogram using DWT with Symlet wavelet. The proposed procedures by using Symlet wavelet implementation to extract MRI images feature is described as the following:

* Menu from command.

* Select the wavelet 2-D toolbox of MATLAB.

* Load MRI brain images.

* Decomposition MRI brain images Sym2.

* Set several levels (2-level) .

* Set an analysis image.

* Detect the Histogram of MRI images.

* Load statistical analysis for the original image.

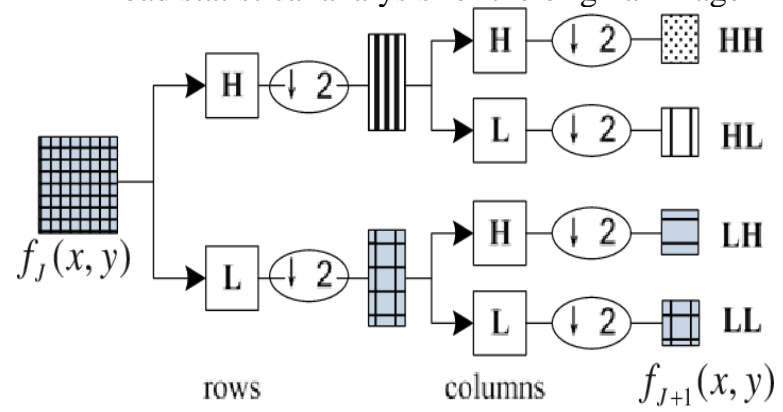

Fig. 4: Decomposition-tree-of-an-image-with-2D-DWT.

3.4.2 Texture features by gray level co-occurrence matrix (GLCM)

The texture is an essential feature for the analysis of several categories of images [26,27] because it gives a good reference of image information [26], biomedical image analysis, remote sensing, and automated inspection of traditional texture analysis applications [28]. Texture evaluates attempts to measure the intuitive properties defined by terms such as coarse, smooth, velvety, or rough as a feature of spatial variation in pixel intensity. Simple texture features can be determined by calculating statistical characteristics, such as mean and variance from the image's gray level histogram. However, the quality of this kind of first-order statistics is generally poor. Calculated secondorder gray - level statistics utilizing gray-level co-occurrence matrices (GLCM) and described fourteen statistical measurements for texture from images [28]. In this work, the function used (graycomatrix) [29] in MATLAB generates (GLCM) gives information on texture patterning and cooccurrence used to compute textural characteristics of the texture. The GLCM features describe the pixels of an image 
by computing how many pixels sets of particular values and spatial configuration happen in an image, generating a GLCM, and then generating statistical measurements from this matrix [27].

Algorithm: For calculating GLCM measures for each pixel: Step1: Read an image of the MRI input.

Step2: Convert the type of data to double,

Step3: Extract from the input image an $8 \times 8$ matrix image.

Step4: Calculate the amount of the texture that co-occurred.

Step5: Estimate the parameters of the texture for the image obtained.

Step6: Repeat step 3,4and 5 by shifting the window until the picture finish.

Step7: View parameters of different texture by normalizing them.

The texture extract features from the GLCM matrix are contrast, correlation, energy, smoothness, homogeneity, and additional IDM. Added features from this matrix were also derived which are: mean, standard deviation, entropy, variance, kurtosis, skewness [27,28].

\subsubsection{Area}

The actual number of pixels returned as a scale in the region. (This value may vary significantly from the value returned by the (beware) function in MATLAB, which weights different pixel patterns differently) [30].

\subsection{Tumor classification stage}

The system consists of two panels: the feature extraction stage (based on preprocessing and post-processing techniques) and the classification stage (based on artificial intelligence algorithms and SVM).

\subsubsection{Artificial neural network structure}

An artificial neural network (ANN) is a machine learning or a computer model that depends on biological neural systems [31]. An ANN includes several basic groups called "neurons" or artificial neurons that are interlinked and work in parallel with processing information, known as parallel distributed processing systems or connecting systems [32]. In this work, the feed-forward backpropagation network (FFBPN) used, which is one of the kinds of artificial neural networks due to the ease of use, has a lot of learning algorithms that can use in the training of the multi-layer neural network.

Setting the neural network as the following:

* Open (nn tool) package from command of MATLAB.

* Import the input layer consists of 13 neurons that are reliant on categorical variables and the number features extracted using a gray-level co-occurrence matrix (GLCM) representing data input. (from the workspace)

* The import output layer consist of one neuron represents the target data vector. (from the workspace)

* Set one hidden layer consist of 3 neurons by using Trial and error

* Set the main Training parameters used in the network for FFBPN.

* Set activation function tensing (sigmoid)

- Minimum gradient threshold of $0.1 \times 10-10$.

- The maximum number of epochs 1000.

- Sum-squared error goal of 0.001 .

- Spread constant 1.

- Maximum time to traininfinity.
- fail-max 10 times to validation check.

- Run to train network.

\subsubsection{Support vector machine}

Vladimir N. Vapnik contributed a support vector machine algorithm and its modern model was designed by Cortes and Vapnik in 1993 [33]. SVM is a supervised machine learning algorithm used to classify data for different classes based on a separating hyperplane. Although there two main types of SVM classifications: linear and non-linear [34]. The input vectors are assigned to the feature vector using the kernel function that directly calculates the dot product in the feature space. The hyper-plane is formed in a dimensional space separated into two classes, which Optimizes the distance between each other and closes the training sets. This hyperplane is used as a basis for classifying vectors of unknown objects (testing objects).

The training sample is $\left\{\left(x_{i}, y_{i}\right)\right\} N i=1$, where $x_{i}$ is represents the set of inputs for the $i^{\text {th }}$ instance and $y_{i}$ represents the output classes (target), which is represented by $y i=+1$ or -1 and each refers to the linearly separable point class, as shown in Fig. 5. The hyper-plane equation that separates is [35]:

$\mathrm{W}^{\mathrm{T}} \mathrm{X}+\mathrm{b}=0$

Where:

$\mathrm{X}$ : is an input vector.

$\mathrm{W}$ : is a modifiable weight vector.

b: is a bias.

$W^{T} X_{i}+b \geq 0 \quad$ For $Y_{i}=+1$

$W^{T} X_{i}+b \leq 0 \quad$ For $Y_{i}=-1$

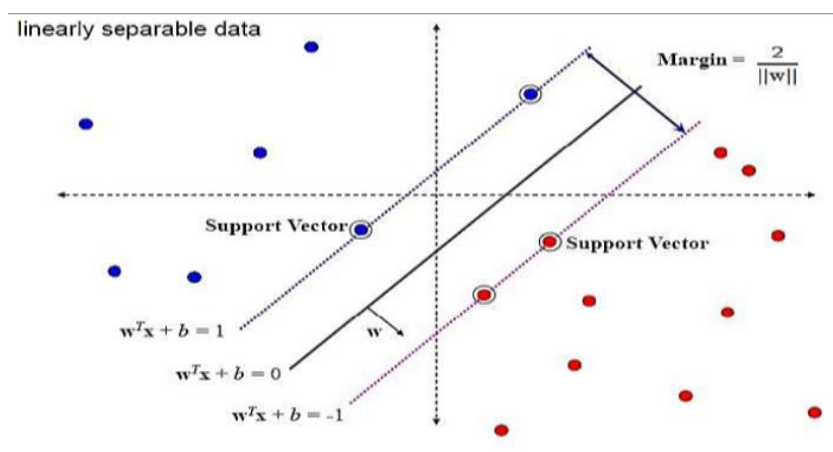

Fig. 5. Support Vector Machine.

The case of the non-linear samples, the distribution of samples is irregular, so that SVM can determine the limitations of non-linear decisions by using kernel function and using two phases. The first phase is the use of the kernel equation that transforms the coming input into the higher dimensional space, And the second phase of the new location searches for a linear hyperplane to classify the samples [34]. There are several types of kernel function below [33] :

1. Radial basis function (RBF)

$k\left(x, x^{\prime}\right)=\exp \left(-\left\|x-x^{\prime}\right\| 2\right)$

Where $x$ and $x^{\prime}$ are sample vectors and $\left\|x-x^{\prime}\right\| 2$ is a Euclidean distance between sample vectors.

2. Linear kernel

$y k=f\left(x, x^{\prime}\right)$

Where $x$ and $x^{\prime}$ are sample vectors where $y k$ is the linear kernel which is a function of linear vectors. 
3. Polynomial kernel

$K\left(x, x^{\prime}\right)=\left(1+x \cdot x^{\prime}\right) k$

Where $x$ and $x^{\prime}$ are sample vectors.

\section{RESULTS AND DISCUSSION}

The experimental results provided by the work technique depicted for the segmented outcome and the extracted tumor region are given in Fig. 6. Concerning the variables with high normalized importance such as kurtosis, contrast, entropy, energy, correlation, and all other variables are not reflecting a threshold of classification to categories of brain tumor categories on their own. This is because other factors need to be involved and added to the set of independent variables. Laboratory information may add substantial information about the type of brain tumor. Observation of the brain tumor images suggests that tumors can be characterized by three factors; these are site, size, and shape. Information about these factors may also help to produce more reliable criteria of classification and Fig. 7 shown the training network for FFBPN. Lastly, 13 features are selected for classification. Feature extraction using the GLCM method where classification using GLCM-based features provides classification accuracy. Also, The GLCM method is easy to perform which has been shown to deliver very excellent results in a variety of application fields [36]. The GLCM method has a good processing time and complexity performance [37] compared to the DWT method. Table 1 shows the texture and area features for eight MRI images including cases (normal, benign, and malignant) for the database, after which will be entered as inputs for the works used for the classification of an image brain tumor after the normalization process.
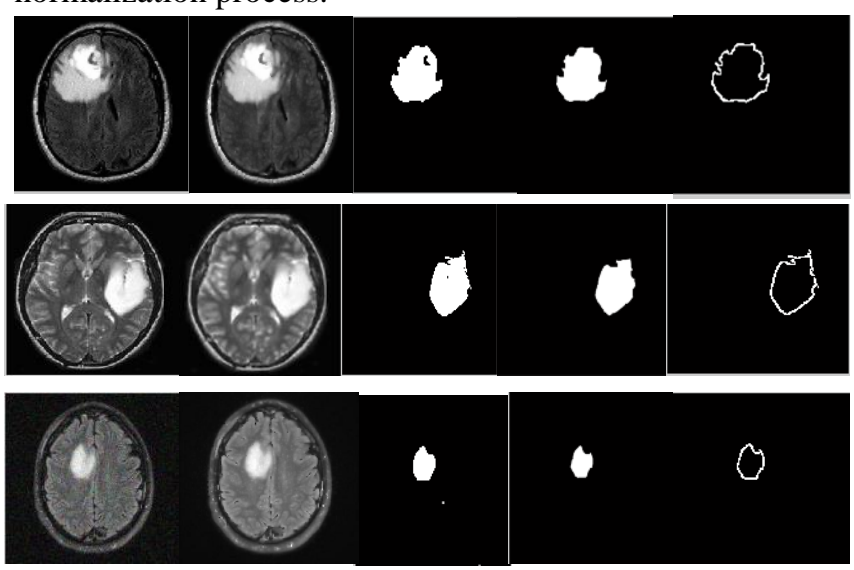

(a)

(b)

(c)

(d)

(e)

Fig. 6: Result of pre-processing AND segmentation (a) Original MRI input image, (b) Anisotropic diffusion filtering, (c) Proposed thresholding segmentation, (d)Eroded and Morphological operation, and (e) Tumor outline.
The typical performance for the FFBPN pre-training using various numbers of neurons in the hidden layer. The 2, 3, and 5 of neurons in the hidden layer has been chosen as the best numbers after employing many training operations for the FFBPN using trial and error method. The 3 neurons were obtained as an optimal number used for the hidden layer. The FFBPN gave $99.93 \%$ accuracy for the training dataset, while $99.99 \%$ and $76.23 \%$ accuracy was recorded for testing and validation datasets respectively. So the unanimous accuracy of the overall method becomes $96.25 \%$. The given accuracy is observed only for 31 epochs. Fig. 8and Fig. 9 shows the system performance of our suggested methodology.

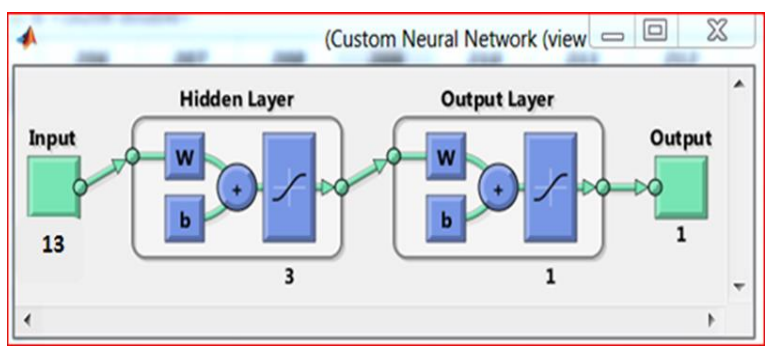

Fig. 7: Topology structure of FFBPN for training network. In this work, using the linear kernel function for transformation. The corresponding performance of the SVM classification is used by the kernel function. The results are shown in the command window of the MATLAB for brain image it is benign or malignant (normal or abnormal) as shown in Fig. 10.

\section{Performance Analysis And Comparison}

The training dataset images for which the extracted features have been practiced using the classification process classifier (FFBPN \& SVM), while the test dataset is not trained using the FFBPN \& SVM classifier, mostly the statistical and textural features have been extracted. The precision of the training and testing images were analyzed by comparison focused on the classification of (normal, benign, and malignant) tumors cells for FFBPN, while SVM was centered on the classification of (benign and malignant) tumors cells. The accuracy or classification performance rate is the effectiveness of the relevant classification for the overall amount of classification checks. The output of the algorithm proposed can be computed using predictive values. There are four predictive values: true positive values (TP), true negative values (TN), false-positive values $(\mathrm{FN})$, and false-positive (FP). This was used to measure the efficiency of the work results presented to the MRI images by sensitivity, specificity, and accuracy as shown in equation (13) (14) (15). The calculation for test images (images used for test after the training process) shown in Table 2 for SVM and Table 3 for FFBPN. 
TABLE 1

Textural features for eight of MRI images in addition to the area of the extracted tumor.

\begin{tabular}{|c|c|c|c|c|c|c|c|c|}
\hline Features & Image 1 & Image 2 & Image 3 & Image 4 & Image 5 & Image 6 & Image 7 & Image 8 \\
\hline Mean & 0.0175 & 0.0653 & 0.0524 & 0.0107 & 0.1484 & 0.0061 & 0.0283 & 0.05338 \\
\hline Standard .dev & 0.3486 & 0.6996 & 0.6428 & 0.2802 & 1.0703 & 0.2182 & 0.4631 & 0.64504 \\
\hline Skewness & 18.9631 & 10.5919 & 11.4712 & 23.5019 & 6.8332 & 13.5967 & 15.0402 & 11.0665 \\
\hline Kurtosis & $4.2 \mathrm{E}+02$ & $1.21 \mathrm{E}+02$ & $1.41 \mathrm{E}+02$ & $6.4 \mathrm{E}+02$ & $9.6 \mathrm{E}-01$ & $4.1 \mathrm{E}+02$ & $2.6 \mathrm{E}+02$ & $1.3 \mathrm{E}+02$ \\
\hline Energy & 0.9504 & 0.9040 & 0.9259 & 0.9614 & 0.8519 & 0.9529 & 0.9393 & 0.908 \\
\hline Entropy & 0.2979 & 0.5567 & 0.4597 & 0.2410 & 0.8074 & 0.3047 & 0.3361 & 0.5078 \\
\hline Contrast & 0.3426 & 0.5319 & 0.3161 & 0.2544 & 0.7581 & 0.3512 & 0.4082 & 0.5755 \\
\hline Homogeneity & 0.9845 & 0.9731 & 0.9815 & 0.9876 & 0.9637 & 0.9847 & 0.9812 & 0.9737 \\
\hline Variance & 0.1150 & 0.40696 & 0.3722 & 0.0741 & 0.9009 & 0.0464 & 0.1932 & 0.3387 \\
\hline Correlation & 0.4727 & 0.6474 & 0.7211 & 0.4262 & 0.7322 & 0.2812 & 0.5202 & 0.5796 \\
\hline smoothness & 0.9902 & 0.9974 & 0.9967 & 0.9841 & 0.9988 & 0.9723 & 0.9939 & 0.9968 \\
\hline IDM & 7.5726 & 53.7549 & 10.9712 & 2.1702 & 123.8235 & 2.2627 & 42.4959 & 27.1191 \\
\hline Area & 774.75 & 2957 & 2487.5 & 501.25 & 6898.375 & 299.25 & 1318 & 2533.25 \\
\hline
\end{tabular}

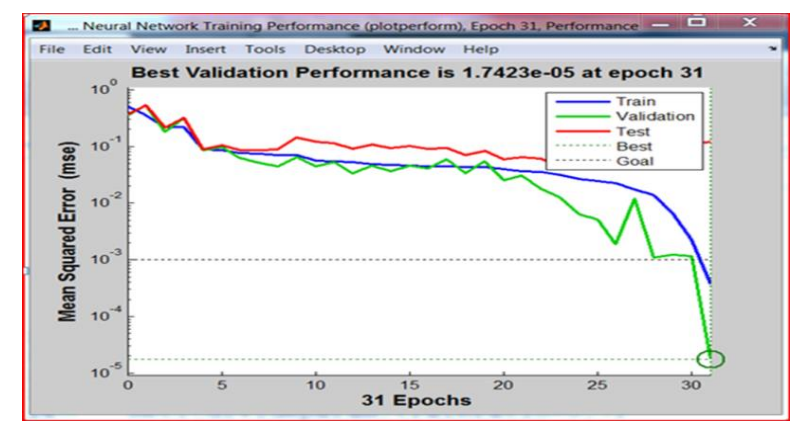

Fig. 8: Typical performance for the FFBPN training

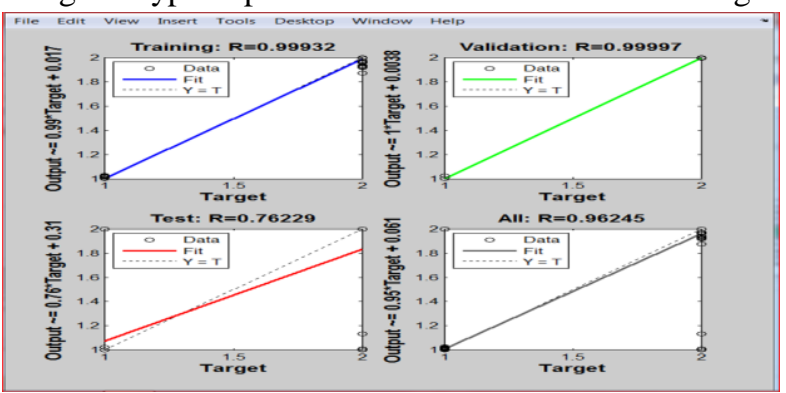

Fig. 9: (Training and validation) and (Testing and overall) regression depicting the relationship between targets and outputs.

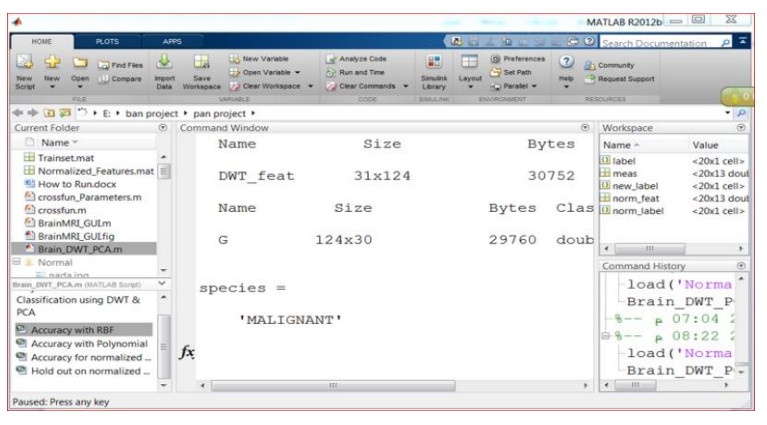

Fig. 10: The result of an SVM classifier

TABLE 2

The experiments dataset in SVM.

\begin{tabular}{|c|c|c|c|}
\hline Cases & Benign & Malignant & Total \\
\hline Benign & $42 \mathrm{TP}$ & $1 \mathrm{FP}$ & 43 \\
\hline Malignant & $6 \mathrm{FN}$ & $51 \mathrm{TN}$ & 57 \\
\hline Total & 48 & 52 & 100 \\
\hline
\end{tabular}


TABLE 3

The experiments dataset in FFBPN.

\begin{tabular}{|c|c|c|c|c|}
\hline Cases & Normal & Benign & Malignant & Total \\
\hline Normal & 36 & 0 & 0 & 36 \\
\hline Benign & 1 & 29 & 2 & 32 \\
\hline Malignant & 0 & 0 & 32 & 32 \\
\hline Total & 37 & 29 & 34 & 100 \\
\hline
\end{tabular}

Sensitivity $=\frac{T P}{T P+F N} \times 100$

Specificity $(\%)=\frac{T N}{F P+T N} \times 100$

Accuracy $(\%)=\frac{\text { Number of correct data }}{\text { Number of all data }} \times 100$

The results of FFBPN and SVM classification performance where SVM classifier gave accuracy $93 \%$ while, FFBPN gave $97 \%$ using the same features as input in them. FFBPN gave the best result for the classification MRI images for brain tumors as shown in Table 4. In this section also, presents a performance comparison of the existing method with the results of the previous works in images processing filed taking from MRI for classifying brain tumor based on different algorithms so higher accuracy of brain tumor classification is the plan of each researcher that tried to stratify it as shown in Table 5.

TABLE 4

FFBPNN and SVM classification performance.

\begin{tabular}{|c|c|c|c|}
\begin{tabular}{|c|c|} 
Classifier \\
type
\end{tabular} & Sensitivity & Specificity & Accuracy \\
\hline SVM & $87.5 \%$ & $98.08 \%$ & $\mathbf{9 3} \%$ \\
\hline FFBPN & $97.39 \%$ & 98.78 & $\mathbf{9 7 \%}$ \\
\hline
\end{tabular}

\section{Conclusions}

This work aimed to submit an Automated algorithm for detecting brain tumors from MRI images by Artificial Neural Networks and SVM. The data collected 308 images and prepared by pre-processing and post-processing processes to make it suitable for detection. Analysis of statistical features has been used to extract features from images; attributes calculated from Graycomatrix features dependent on the (GLCM) of images. In the case of artificial neural networks, the feed-forward backpropagation network with supervised learning has been used to classify the images as normal, benign, and malignant. And all the right features were used as input variables for the FFBPN, then the network was trained and its performance is measured. Finally; the proposed algorithm, the FFBPN, gives the best results by detecting and classifying brain tumors according to the extraction feature achieved results with a precision of $97 \%$ while the SVM has low accuracy. The proposed system effectively classifies the images of the brain tumor of the MRI.
TABLE 5

Comparison result with previous works of detection and classification of brain tumors.

\begin{tabular}{|c|c|c|c|}
\hline Authors & Features & $\begin{array}{c}\text { Classification } \\
\text { Algorithm }\end{array}$ & Accuracy \\
\hline $2014[38]$ & $\begin{array}{c}\text { Extracted } 4 \\
\text { textural } \\
\text { features by } \\
\text { GLCM } \\
\text { method }\end{array}$ & KNN & $97.3 \%$ \\
\hline 2017 [7] & $\begin{array}{c}\text { Extracted } 4 \\
\text { textural } \\
\text { features by } \\
\text { GLCM } \\
\text { method }\end{array}$ & $\begin{array}{l}\text { SVM-based } \\
\text { classification }\end{array}$ & $95 \%$ \\
\hline 2018 [8] & $\begin{array}{l}\text { Extracted } 5 \\
\text { textural } \\
\text { features by } \\
\text { GLCM and } \\
\text { DWT }\end{array}$ & PNN classifier & $\begin{array}{l}\text { Nearly } 100 \% \\
\text { was achieved } \\
\text { for trained } \\
\text { and } 95 \% \text { for } \\
\text { tested }\end{array}$ \\
\hline 2019 [39] & $\begin{array}{c}\text { Extracted } 9 \\
\text { features by } \\
\text { DWT } \\
\text { technique }\end{array}$ & FF-ANN & $95.48 \%$ \\
\hline 2019 [9] & $\begin{array}{c}9 \text { features by } \\
\text { GLCM } \\
\text { method }\end{array}$ & $\begin{array}{l}\text { SVM, FFNN } \\
\text { and ELM }\end{array}$ & $\begin{array}{c}92.99 \%, 89.41 \% \\
\text { and } 90.20 \%\end{array}$ \\
\hline $2020[10]$ & $\begin{array}{l}\text { Extracted } 5 \\
\text { textural } \\
\text { features by } \\
\text { GLCM }\end{array}$ & SVM & $90 \%$ \\
\hline $2020[40]$ & $\begin{array}{c}3 \text { kinds of } \\
\text { features are } \\
\text { extracted as } \\
\text { shape } \\
\text { features- } \\
\text { intensity } \\
\text { features and } \\
\text { kurtosis } \\
\text { texture } \\
\text { features by } \\
\text { DWT }\end{array}$ & $\begin{array}{l}\text { KNN and } \\
\text { SVM }\end{array}$ & $\begin{array}{c}85.2 \%, 90.75 \% \\
\text { with } \\
\text { smote sampling } \\
\text { disabled and } \\
88.75 \% \\
\text {,93.32\%y with } \\
\text { smote sampling } \\
\text { enabled }\end{array}$ \\
\hline $\begin{array}{c}\text { Propose } \\
\text { Algorithm }\end{array}$ & $\begin{array}{c}\text { Extracted } 13 \\
\text { textural } \\
\text { features by } \\
\text { GLCM } \\
\text { method }\end{array}$ & $\begin{array}{l}\text { SVM and } \\
\text { FFBPN }\end{array}$ & $93 \%$ and $97 \%$ \\
\hline
\end{tabular}

\section{SUGGESTIONS FOR FUTURE WORK}

This work can be extended in the future according to expansion the data base to include the other type of MRI images, developing the algorithm to detect and diagnoses brain tumor to the 3D images MRI.Using the algorithm to extend to the analysis of other medical images, such as CT and PET images. Attempt to contact other laboratories on the internet carrying out similar research to share data and work together to establish to open test database. 


\section{CONFLICT OF INTEREST}

The authors have no conflict of relevant interest to this article.

\section{REFERENCES}

[1] Young, RM; Jamshidi, A; Davis, G; Sherman, J. H. "Current trends in the surgical management and treatment of adult glioblastoma", Annals of Translational Medicine, Vol. 3, Issue 9, June 2015.

[2] Umar, A. A., and S. M. Atabo. "A review of imaging techniques in scientific research/clinical diagnosis", MOJ Anat. \& Physiol., Vol. 6, Issue 5, 2019, pp. 175183.

[3] Khodamoradi F., et al., "The incidence and mortality of brain and central nervous system cancer and their relationship with human development index in the world", World Cancer Res J, Vol. 4, 2017.

[4] Almutrafi Amna, et al., "The Epidemiology of Primary Central Nervous System Tumors at the National Neurologic Institute in Saudi Arabia: A Ten-Year Single-Institution Study", Journal of Cancer Epidemiology, 2020.

[5] Naveena H., Shreedhara K., and Mohamed Rafi, "Detection and Classification of Brain Tumor using BPN and PNN Artificial Neural Network Algorithms", International Journal of Computer Science and Mobile Computing. IJCSMC, Vol. 4, Issue. 4, April 2015, pp.782 - 789 .

[6] Chaddad Ahmad and Tanougast Camel "Quantitative evaluation of robust skull stripping and tumor detection applied to axial MR images", Brain Informatics, Vol. 3, Issue 1, 2016, pp. 53-61.

[7] Bahadure, Nilesh Bhaskarrao et al., "Image analysis for MRI based brain tumor detection and feature extraction using biologically inspired BWT and SVM", International journal of biomedical imaging, 2017.

[8] Shree, N. Varuna; Kumar, T. N. R., "Identification and classification of brain tumor MRI images with feature extraction using DWT and probabilistic neural network", Brain informatics, Vol. 5, Issue 1, 2018.

[9] Parasuraman Kumar, B. VijayKumar, "Brain Tumor MRI Segmentation and Classification Using Ensemble Classifier", International Journal of Recent Technology and Engineering (IJRTE), ISSN: 2277-3878, Vol. 8, Issue 1S4, June 2019, pp. 244-152.

[10] Ahsanullah Umary, Harpreet Kaur., "Automatic Brain Tumour Diagnosis and Segmentation: based on SVM Algorithm", International Journal of Innovative Technology and Exploring Engineering (IJITEE)ISSN: 2278-3075, Volume-9 Issue-6, April 2020, pp. 1079. 1084

[11] Padmanaban, Sriramakrishnan \& Thiruvenkadam, Kalaiselvi \& T., Padmapriya \& KUMAR, RAM \& Nagarajan, Kalaichelvi. "An Medical Image File Formats and Digital Image Conversion". International Journal of Engineering and Advanced Technology. Vol9 Issue-1S4. 2020. 74-78.
[12] J. S. Ignacio, S. J. Buso, and W. A. Monteiro, "processing and analysis of digital images: How to ensure the quality of data captured?", International Journal of Recent advances in Mechanical Engineering (IJMECH), Vol. 2, No. 2, 2013.

[13] Larobina, Michele; Murino, Loredana. "Medical image file formats". Journal of digital imaging, 2014, Vol. 27, Issue 2, pp. 200-206.

[14] Convert RGB image or colormap to grayscale MATLAB ...

https://www.mathworks.com/help/matlab/ref/rgb2gray. html

[15] Contrast Adjustment-MATLAB \& Simulink - Math works.

https://www.mathworks.com/discovery/imageenhancement.html.

[16] K, S \& Kalitkar, Kishan \& Subba, G \& Rao,. (2015)." A Review on Noise Reduction Methods for Brain MRI Images". SPACES-2015, Dept. of ECE, K L UNIVERSITY. 10.1109/SPACES.2015.7058284.

[17] P. Perona and J. Malik. "Scale-space and edge detection using anisotropic diffusion". IEEE Transactions on Pattern Analysis and Machine Intelligence, Vol. 12, Issue 7, pp. 629-639, 1990.

[18] Reddy, Mummadi Gowthami; REDDY, Palagiri Veera Narayana; REDDY, Patil Ramana. Medical Image Fusion Using Integrated Guided Nonlinear Anisotropic Filtering with Image Statistics. International Journal of Intelligent Engineering and Systems, Vol.13, No.1, 2020

[19] Weickert, J. "Anisotropic diffusion in image processing". Stuttgart: Teubner, Vol. 1 (1998). pp. 5960.

[20]Zhan Yantong; Zhang Guoying. "An improved OTSU algorithm using histogram accumulation moment for ore segmentation". Symmetry, 2019, 11.3: 431.

[21] Cao, Xinhua, et al. A robust parameter-free thresholding method for image segmentation. IEEE Access, 2018, 7: 3448-3458.

[22] Qidwai, Uvais, and Chi-hau Chen. "Digital image processing: an algorithmic approach with MATLAB". CRC press, 2009.

[23] Ilhan Umit; Ilhan Ahmet. "Brain tumor segmentation based on a new threshold approach". Procedia computer science, 2017, 120: 580-587.

[24] Icke, Guzide, Kamarthi, V. Sagar, "Feature extraction through discrete wavelet transform coefficients", Intelligent Systems in Design and Manufacturing VI, Proceedings of the SPIE, Volume 5999, pp. 27-35, 2005.

[25] Fouad, Amal; MOFTAH, Hossam M.; HEFNY, Hesham A. Brain Diagnoses Detection Using Whale Optimization Algorithm Based on Ensemble Learning Classifier. International Journal of Intelligent Engineering and Systems, Vol.13, No.2, 2020

[26] Mahmood, Faleh H.; Abbas, Wafaa A. Texture Features Analysis using Gray Level Co-occurrence Matrix for Abnormality Detection in Chest CT Images. Iraqi Journal of Science, Vol. 57, Issue 1A, pp. 279-288, 2016. 
[27] Armi Laleh Fekri-Ershad Shervan, "Texture image analysis and texture classification methods-A review", arXiv preprint arXiv:1904.06554, 2019.

[28]Zulpe N., Pawar V., "GLCM Textural Features for Brain Tumor Classification", International Journal of Computer Science Issues, Vol. 9, Issue 3, No 3, 2012.

[29] Texture Analysis Using the Gray-Level CoMathWorks.

https://www.mathworks.com/help/images/textureanalysis-using-the-gray-level-co-occurrence-matrixglcm.html.

[30] Area of objects in binary image - MATLAB bwarea. https://www.mathworks.com/help/images/ref/bwarea.ht $\mathrm{ml}$

[31]Dr. Adel M. Dakhil, "Short Term Load Forecasting Based Artificial Neural Network", Iraq Journal for Electrical and Electronic Engineering, Vol. 10, No. 1, 2014.

[32] K. L. Du, M. N. Swamy, "Neural Network in Soft Computing”, Springer, 2006.

[33] Singh, Khushboo; Verma, Satya. Detecting Brain Mri Anomalies By Using Svm Classification. International Journal of Engineering Research and Applications (IJERA) Vol. 2, pp. 724-726, 2012.

[34] M.S. Uzer, N. Yilmaz and O. Inan,"Feature Selection Method Based on Artificial Bee Colony Algorithm and Support Vector Machines F=for Medical Datasets Classification". The Scientific World Journal, 2013.

[35]F. Melgani And L. Bruzzone, "Classification of Hyper spectral Remote Sensing Images With Support Vector Machines", IEEE Transactions on Geoscience and Remote Sensing, Vol. 42, Issue 8, pp.1778-1790, 2004.

[36]P. Mohanaiah, P. Sathyanarayana, and L. GuruKumar."Image texture feature extraction using GLCM approach". International Journal of Scientific and Research Publication., vol. 3, no. 5, p. 1, 2013.

[37] M. Mehri, M., Héroux, P. Gomez-Krämer, and R. Mullot."Texture feature benchmarking and evaluation for historical document image analysis". Int. J. Document Anal. Recognit., vol. 20, no. 1, pp. 1-35, Mar. 2017

[38] Chitari Amruta, BAG V. V., "Detection of brain tumour using classification algorithm", Int. J. Invent. Comput. Sci. Eng, Vol. 1, pp. 2348-3539, 2014.

[39]Zahid Ullah, Su-Hyun Lee, Muhammad Fayaz, "Enhanced feature extraction technique for brain MRI classification based on Haar wavelet and statistical moments", International Journal of Advanced and Applied Sciences, Vol. 6, Issue 7, 2019, pp. 89-98.

[40] Latha, r. S., et al., "Brain Tumor Classification Using SVM and KNN Models For Smote Based MRI Images", Journal of Critical Reviews, Vol. 7, Issue 12, pp. 1-4, 2020 . 